\title{
High concordance between next generation sequencing and single-nucleotide polymorphism array in preimplantation genetic testing for aneuploid
}

\section{Zhanhui Ou}

Guangzhou Women and Children's Medical Center

\section{Zhiheng Chen}

Guangzhou Women and Children's Medical Center

\section{Yu Deng}

Guangzhou Women and Children's Medical Center

\section{Minna Yin}

Guangzhou Women and Children's Medical Center

Ling Sun ( $\nabla$ sunling6299@163.com )

Guangzhou Women and Children's Medical Center

\section{Research article}

Keywords: Chromosomal abnormalities, Next generation sequencing, Preimplantation genetic testing for aneuploid; Preimplantation genetic testing for chromosomal structural rearrangement, Single-nucleotide polymorphism array

Posted Date: October 7th, 2020

DOI: https://doi.org/10.21203/rs.3.rs-85962/v1

License: (c) (i) This work is licensed under a Creative Commons Attribution 4.0 International License. Read Full License 


\section{Abstract}

Background: Single-nucleotide polymorphism array (SNP array) and next generation sequencing (NGS) in detecting chromosome aneuploidy are widely used in clinical work.

Aims: To compare the concordance between NGS and SNP array in 67 embryos (from 23 couples).

Methods: In the first part of the study, 28 blastocysts with unknown ploidy were both analyzed with NGS and SNP array. While in the second part, 39 with normal ploidy detected by NGS were re-analyzed with SNP array.

Results: In the first part of the study, the concordance rate between NGS and SNP array was $92.9 \%$ (26/28). Among the 28 blastocysts, 18 were abnormal and 10 blastocysts were with normal ploidy status when analyzed by NGS. Among the 18 abnormal blastocysts, two blastocysts were with low level of mosaicism as analyzed by NGS, but euploid with SNP array. In the second part, concordance rate between NGS and SNP array was 100\% (39/39). At last, one couple had no blastocyst to transfer. The other 22 couples were transferred with single blastocyst. Among them, two couples suffered abortions before 12 weeks, and the karyotype of villus was normal. One couple with only 1 normal blastocyst failed to conceive after the transfer. In total nineteen couples had healthy babies born.

Conclusions: There was a high concordance rate between NGS and SNP array. But NGS was also able to detect mosaicism sensitively. Hence, using NGS for PGT-A may increase the chances of having a healthy and live newborn child.

\section{Introduction}

Chromosomal abnormalities are widespread in human embryos produced in vitro [1]. Incidences of abnormalities increases dramatically in embryos with advanced maternal age and with one of the parents with balanced reciprocal chromosomal translocations [2-5]. Chromosomal abnormalities are one of the main reasons for spontaneous abortions [6,7], and repeated implantation failures [8,9]. Furthermore, embryos from carriers of balanced translocations are at a particularly high risk of chromosomal abnormalities [10,11].

Selecting a normal or balanced embryo for transfer increases the chances of having a healthy and live newborn child. Preimplantation genetic testing for aneuploid (PGT-A) and preimplantation genetic testing for chromosomal structural rearrangement (PGT-SR) were widely used to screen embryos' genetic condition prior to transfer for advanced maternal age couples, recurrent implantation failure, recurrent miscarriage patients and chromosome structure abnormality carrier patients [12]. It has been found that transfer of PGT-screened normal blastocysts could significantly increase implantation and reduce pregnancy loss [13-15]. Hence, these techniques were widely used in chromosomal and genetic disease diagnosis. 
With the development of genome-wide technologies, the technique of array comparative genomic hybridization (aCGH) and single nucleotide polymorphism (SNP) microarrays are extensively applied to reduce the risk of aneuploidy $[16,17]$. However, NGS is becoming more and more popular in clinic, due to the fact that approximately $1 \mathrm{Mb}$ of deletions or duplications and low level of mosaicism in chromosomes could be detected via NGS. It can decrease cost with high throughput compared with aCGH and SNP arrays $[18,19]$. However, there are few studies about comparison between SNP array and NGS in detecting chromosome aneuploidy in the same blastocysts.

The purpose of this study was to compare SNP array and NGS in detecting chromosome aneuploidy. Hence, 67 embryos obtained from preimplantation genetic testing for aneuploid, reciprocal translocation or Robertsonian translocation carriers were adopted for the retrospective studies. The clinical outcomes, including clinical pregnancy and miscarriages, were also recorded to evaluate the use of NGS in clinic.

\section{Materials And Methods}

\section{Ethics}

This study was approved by the Reproductive Medical Ethics Committee of Guangzhou Women and Children's Hospital. Written informed consent was obtained from each couple.

\section{Embryo sources}

In this study, 23 couples were undergoing PGT-A/PGT-SR in our center from March 2017 to June 2018. The indications of PGT-SR are reciprocal translocation, Robertsonian translocation carriers and chromosome inversion. Indications of PGT-A are sex chromosome aneuploidies.

In the first part of the study, 28 blastocysts from 5 couples were detected by both NGS and SNP array. In the second part, 115 blastocysts from 18 couples were only detected by NGS firstly. Among them, 39 blastocysts were normal. These blastocysts were detected by SNP array again (Fig. 1).

\section{Embryo culture and biopsy}

Intracytoplasmic sperm injection (ICSI) were performed after the oocytes were retrieved, sequence culture was enrolled by G1/G2 (Vitrolife, Sweden). Biopsy was performed on day 5 or day 6 according to the blastocyst grade [20].

All of the blastocysts were subjected to trophectoderm-cell-biopsy by laser and 5-10 TE cells were biopsied. After biopsied, blastocysts were cryopreserved as planned using vitrification technique according to the manufacturer's protocol (ARSCI Inc, Canada) and stored in liquid nitrogen.

\section{Whole genome amplification}

The multiple displacement amplification (MDA) DNA amplification system was used for whole genome amplification (WGA). Briefly, REPLI-g Single Cell Kit (QIAGEN, Germany) was used for single cell 
amplification, single cell was seeding in $2.5 \mu \mathrm{l}$ phosphate-buffered saline, and then was lysised using $3 \mu \mathrm{l}$ DTT and $33 \mu \mathrm{l}$ DLB in incubator for $10 \mathrm{~min}$ at $65^{\circ} \mathrm{C}$. After incubation, $3 \mu \mathrm{l}$ Stop Solution was added in the mix. The amplification mix was prepared and $40 \mu$ including $2 \mu \mathrm{l}$ REPLI-g DNA polymerase and $29 \mu \mathrm{l}$ REPLI-g Reaction Buffer was added to each tube. Then the mixture was incubated in incubator for $8 \mathrm{~h}$ at $30^{\circ} \mathrm{C}$ following $65^{\circ} \mathrm{C}$ for 3 min to inactivate the REPLI-g DNA polymerase.

\section{NGS protocol}

The Illumina MiSeq platform was used in this next-generation sequencing (NGS), and the amplified genome of each blastocyst's TE cells were sequenced at an approximate $0.01 \times$ genome depth. An oninstrument computer performs primary and secondary data analysis to align the reads to a reference genome. We sequenced a total of approximately 36 million bases, obtaining average genome coverage of $1.2 \%$ for each blastocyst's TE sample. PGXcloud cloud server (available at http://www.pgxcloud.com/) was used to analyze the chromosomal copy number variants (CNVs) (Jabrehoo, China). All results were examined by two independent laboratory technicians to minimize uncertainty and variable results. In the case of discrepancies in opinion, a consensus was reached after the third technician discussion. The structural variants detected is $>4 \mathrm{Mb}$ and the level of mosaicism detected is $>30 \%$ in our analysis.

\section{SNP array}

All the procedures were performed according the instruction of Illumina human SNP array. Briefly, DNA were hybridized with the Human Cyto-12 BeadChip (Illumina), which contains approximately 300,000 SNPs with an average distance of $9.7 \mathrm{~kb}$. The bead chips were subjected to immunostaining, followed by stringent washes to remove unhybridized and non-specifically hybridized DNA. Subsequently, the bead chips were scanned using an Illumina iScan Bead Array Reader (Illumina). The scanning results were processed using the B allele frequency and log R ratio using Illumina Genome Studio software (Illumina) to analyze the copy number of the chromosomes according the instruction of Illumina human SNP array.

\section{Results}

\section{Concordance between NGS and SNP array in the first part}

In the first part, the concordance between NGS and SNP array was $92.9 \%(26 / 28)$. Among the 28 blastocysts, 16 blastocysts were abnormal and 12 blastocysts were with normal ploidy status detected by NGS. However, compared with the result from NGS, that 14 blastocysts were abnormal and 14 blastocysts were normal analyzed by SNP array. Two blastocysts were completely discordant between NGS and SNP. The abnormality detected by NGS showed those two blastocysts were with low level of mosaicism, while normal when detected by SNP array (Table 1, embryo number 1 and 2, Fig. 2). Among the other 14 abnormal blastocysts, one blastocyst was with discordant results, because the mosaicism could be only detected by NGS (Table 1, embryo number 3, Fig. 3 ). 


\begin{tabular}{lll}
\hline $\begin{array}{l}\text { Embryo } \\
\text { Number }\end{array}$ & NGS result & SNP array result \\
\hline 1 & $46, \mathrm{XN}, \operatorname{mos} \operatorname{dup}(6)(40 \%), \operatorname{mos} \operatorname{dup}(13)(38 \%), \operatorname{mos} \operatorname{dup}(14)(31 \%), \operatorname{mos} \operatorname{dup}(15)(40 \%), \operatorname{mos}$ & $46, \mathrm{XN}$ \\
& $\operatorname{dup}(16)(40 \%)$ & $46, \mathrm{XN}$ \\
2 & $46, \mathrm{XN}, \operatorname{mos} \operatorname{dup}(13)(44 \%)$ & $44, \mathrm{XN}, \operatorname{del}(6), \operatorname{dup}(12), \operatorname{dup}(14), \operatorname{del}(15)$ \\
\hline
\end{tabular}

46,XN : 46,XX or 46,XY. Mos: mosaic. Dup: duplication. Del: deletion. \%: The level of mosaicism.

\section{Concordance between NGS and SNP array in the second part}

One hundred and fifteen blastocysts from 18 couples were firstly detected by NGS. Among them, 39 blastocysts were normal. These blastocysts were detected by SNP array again. And the concordance rete between NGS and SNP array was 100\% (39/39) (Fig. 1).

\section{The clinical outcome}

One couple has no normal blastocyst (only two blastocysts) for transfer, because all of their blastocysts were with abnormal karyotype. Aother 22 couples were transferred with single blastocyst. Among them, two couples miscarried at 8- and 11-week's gestation respectively, and the abortion villus show normal karyotype detected by NGS. One couple with only 1 normal blastocyst failed to conceive after transfer. Nineteen couples had healthy babies born, and their prenatal diagnosis results and karyotype analysis after the delivery were concordant with the PGT results.

\section{Discussion}

TE biopsy combined with NGS for PGT-A/PGT-SR is now widely used in clinical work around the world. It had been proven to increase the pregnancy rate of patients with chromosome structure abnormality carrier, advanced maternal age and recurrent miscarriage patients [21,22]. NGS technique is showed to have more advantages than SNP array, including the low price that can support its extensive use in selecting the normal embryo for implantation. NGS is also able to detect mosaicism sensitively $[21,23]$. Hence, using NGS for PGT-A may increase the chances of having a healthy and live newborn child, it also lessens the stress of abortion [24-26]. In our study, there is a high concordance in detecting the blastocyst ploidies between NGS and SNP array except the low level of mosaic embryos. Three blastocysts (2 blastocysts only with mosaicism and 1 blastocyst combine with other abnormality) were with mosaicism analyzed by NGS, which could not be found by SNP array. There is paradox about the mosaicism as the couples can still have healthy babies born after transferring mosaic embryos. Studies suggested that the level of mosaicism identified in the original TE biopsy was a predictor of miscarriage [27]. However, there was also a study which reported that mosaic embryos with low aneuploidy percentage had less chances to cause a miscarriage and could lead to a healthy birth [24]. Therefore, transferring mosaic blastocysts is still controversial on clinical risk management and/or difficult counselling perspectives.

Both SNP arrays and NGS platforms have potential strengths and weaknesses. SNPs are a genotyping array that can detect uniparental disomic, identify the parental origin of chromosome abnormalities and 
detect triploid [28]. NGS can screen approximately $1 \mathrm{Mb}$ of deletions or duplications in chromosomes and have high sensitivity to detect low level of mosaicism. Hence, NGS would find more abnormality than other methods and improve the implantation. Tan compared the NGS to SNP array in PGT-A. Results showed that NGS was able to detect some segmental imbalances that may be omitted by SNP array. So that the potential risks of false-negative results can be avoided by the NGS. Although the clinical outcome measures of the NGS cycles were the same as SNP array, it seems that the implantation and clinical pregnancy rate in NGS were higher than the rate in SNP array [21].

In this study, according to the NGS and SNP array result, 23 blastocysts were transferred into the uterus with single blastocyst, and 22 were implanted and developed. The karyotype was normal in the abortion villus or the blood from the newborns. This indicates the accuracy of NGS and SNP array in the PGT. It illustrates that NGS is applicable for the genetically high-risk populations, such as carriers of Robertsonian translocations and reciprocal translocations. However, three mosaic embryos could be detected by NGS, but failed to be confirmed by SNP array. Therefore, with the detection by NGS, embryos with mosaic abnormalities can be selected and will not be used for transfer, this avoids the abortion or newborns with prenatal abnormality caused by embryos with mosaic abnormalities which could not be detected by SNP. In our previous study, some embryos were classified as mosaic in the original analysis by NGS were re-classified as chromosomally balanced after whole blastocyst sequencing. Hence, to maximize the number of embryos available for PGT-SR/A patients, we suggest that embryos with mosaic non-SR chromosomal rearrangement should be stored and considered for transfer after appropriate counseling [29].

There are some limitations in this study. Firstly, there were relatively few mosaic blastocysts in this research, we need more samples for better understanding the embryos with mosaic results. Secondly, all samples in the second part were normal. It could be much better to compare these methods using both normal and abnormal embryos.

\section{Conclusion}

In conclusion, the results achieved in this study demonstrate the reliability of the NGS-based protocol for the detection of chromosome status in embryos and it also able to detect mosaicism sensitively.

\section{Declarations}

Funding: This study was supported by institutional funding of Youth medicine research and development program for Reproductive Medicine from Chinese Medical Association (17020150684, Dr.Ou), Health Science and technology project of Guangzhou (20201A011029, Dr.Ou).

Conflict of Interest: The authors declare that they have no competing interests.

Ethics approval: This study was approved by the Reproductive Medical Ethics Committee of Guangzhou Women and Children's Hospital. 
Consent to participate: Written informed consent was obtained from all individual participants included in the study.

Consent for publication: Additional informed consent was obtained from all individual participants for whom identifying information is included in this article.

\section{References}

1. Vanneste $E$, Voet T, Le Caignec C, Ampe M, Konings P, Melotte C, Debrock S, Amyere M, Vikkula M, Schuit F, Fryns JP, Verbeke G, D'Hooghe T, Moreau Y, Vermeesch JR (2009) Chromosome instability is common in human cleavage-stage embryos. Nature medicine 15 (5):577-583. doi:10.1038/nm.1924

2. Kuliev A, Cieslak J, Ilkevitch Y, Verlinsky Y (2003) Chromosomal abnormalities in a series of 6,733 human oocytes in preimplantation diagnosis for age-related aneuploidies. Reproductive biomedicine online 6 (1):54-59. doi:10.1016/s1472-6483(10)62055-x

3. Pellestor F, Andreo B, Arnal F, Humeau C, Demaille J (2003) Maternal aging and chromosomal abnormalities: new data drawn from in vitro unfertilized human oocytes. Human genetics 112 (2):195-203. doi:10.1007/s00439-002-0852-x

4. Grati FR, Barlocco A, Grimi B, Milani S, Frascoli G, Di Meco AM, Liuti R, Trotta A, Chinetti S, Dulcetti F, Ruggeri AM, De Toffol S, Clementi M, Maggi F, Simoni G (2010) Chromosome abnormalities investigated by non-invasive prenatal testing account for approximately $50 \%$ of fetal unbalances associated with relevant clinical phenotypes. American journal of medical genetics Part A 152A (6):1434-1442. doi:10.1002/ajmg.a.33370

5. Treff NR, Northrop LE, Kasabwala K, Su J, Levy B, Scott RT, Jr. (2011) Single nucleotide polymorphism microarray-based concurrent screening of 24-chromosome aneuploidy and unbalanced translocations in preimplantation human embryos. Fertility and sterility 95 (5):16061612.e1601-1602. doi:10.1016/j.fertnstert.2010.11.004

6. Kim JW, Lee WS, Yoon TK, Seok HH, Cho JH, Kim YS, Lyu SW, Shim SH (2010) Chromosomal abnormalities in spontaneous abortion after assisted reproductive treatment. BMC medical genetics 11:153. doi:10.1186/1471-2350-11-153

7. Hodes-Wertz B, Grifo J, Ghadir S, Kaplan B, Laskin CA, Glassner M, Munne S (2012) Idiopathic recurrent miscarriage is caused mostly by aneuploid embryos. Fertility and sterility 98 (3):675-680. doi:10.1016/j.fertnstert.2012.05.025

8. Raziel A, Friedler S, Schachter M, Kasterstein E, Strassburger D, Ron-El R (2002) Increased frequency of female partner chromosomal abnormalities in patients with high-order implantation failure after in vitro fertilization. Fertility and sterility 78 (3):515-519. doi:10.1016/s0015-0282(02)03298-3

9. Menasha J, Levy B, Hirschhorn K, Kardon NB (2005) Incidence and spectrum of chromosome abnormalities in spontaneous abortions: new insights from a 12-year study. Genetics in medicine : official journal of the American College of Medical Genetics 7 (4):251-263. doi:10.109701.GIM.0000160075.96707.04 
10. Munne S, Sandalinas M, Escudero T, Fung J, Gianaroli L, Cohen J (2000) Outcome of preimplantation genetic diagnosis of translocations. Fertility and sterility 73 (6):1209-1218. doi:10.1016/s0015-0282(00)00495-7

11. Verlinsky Y, Tur-Kaspa I, Cieslak J, Bernal A, Morris R, Taranissi M, Kaplan B, Kuliev A (2005) Preimplantation testing for chromosomal disorders improves reproductive outcome of poorprognosis patients. Reproductive biomedicine online 11 (2):219-225. doi:10.1016/s14726483(10)60961-3

12. Harper JC, Wilton L, Traeger-Synodinos J, Goossens V, Moutou C, SenGupta SB, Pehlivan Budak T, Renwick P, De Rycke M, Geraedts JP, Harton G (2012) The ESHRE PGD Consortium: 10 years of data collection. Human reproduction update 18 (3):234-247. doi:10.1093/humupd/dmr052

13. Dahdouh EM, Balayla J, Garcia-Velasco JA (2015) Comprehensive chromosome screening improves embryo selection: a meta-analysis. Fertility and sterility 104 (6):1503-1512. doi:10.1016/j.fertnstert.2015.08.038

14. Friedenthal J, Maxwell SM, Munne S, Kramer Y, McCulloh DH, McCaffrey C, Grifo JA (2018) Next generation sequencing for preimplantation genetic screening improves pregnancy outcomes compared with array comparative genomic hybridization in single thawed euploid embryo transfer cycles. Fertility and sterility 109 (4):627-632. doi:10.1016/j.fertnstert.2017.12.017

15. Majumdar G, Majumdar A, Lall M, Verma IC, Upadhyaya KC (2016) Preimplantation genetic screening for all 24 chromosomes by microarray comparative genomic hybridization significantly increases implantation rates and clinical pregnancy rates in patients undergoing in vitro fertilization with poor prognosis. Journal of human reproductive sciences 9 (2):94-100. doi:10.4103/0974-1208.183512

16. Tobler KJ, Brezina PR, Benner AT, Du L, Xu X, Kearns WG (2014) Two different microarray technologies for preimplantation genetic diagnosis and screening, due to reciprocal translocation imbalances, demonstrate equivalent euploidy and clinical pregnancy rates. Journal of assisted reproduction and genetics 31 (7):843-850. doi:10.1007/s10815-014-0230-3

17. Wang L, Cram DS, Shen J, Wang X, Zhang J, Song Z, Xu G, Li N, Fan J, Wang S, Luo Y, Wang J, Yu L, Liu J, Yao Y (2014) Validation of copy number variation sequencing for detecting chromosome imbalances in human preimplantation embryos. Biology of reproduction 91 (2):37. doi:10.1095/biolreprod.114.120576

18. Wells D, Kaur K, Grifo J, Glassner M, Taylor JC, Fragouli E, Munne S (2014) Clinical utilisation of a rapid low-pass whole genome sequencing technique for the diagnosis of aneuploidy in human embryos prior to implantation. Journal of medical genetics 51 (8):553-562. doi:10.1136/jmedgenet2014-102497

19. Huang J, Yan L, Lu S, Zhao N, Xie XS, Qiao J (2016) Validation of a next-generation sequencingbased protocol for 24-chromosome aneuploidy screening of blastocysts. Fertility and sterility 105 (6):1532-1536. doi:10.1016/j.fertnstert.2016.01.040

20. Harton GL, Magli MC, Lundin K, Montag M, Lemmen J, Harper JC, European Society for Human R, Embryology PGDCESIG (2011) ESHRE PGD Consortium/Embryology Special Interest Group--best 
practice guidelines for polar body and embryo biopsy for preimplantation genetic diagnosis/screening (PGD/PGS). Human reproduction 26 (1):41-46. doi:10.1093/humrep/deq265

21. Tan Y, Yin X, Zhang S, Jiang H, Tan K, Li J, Xiong B, Gong F, Zhang C, Pan X, Chen F, Chen S, Gong C, Lu C, Luo K, Gu Y, Zhang X, Wang W, Xu X, Vajta G, Bolund L, Yang H, Lu G, Du Y, Lin G (2014) Clinical outcome of preimplantation genetic diagnosis and screening using next generation sequencing. GigaScience 3 (1):30. doi:10.1007/s10815-015-0450-1 10.1186/2047-217x-3-30

22. Lukaszuk K, Pukszta S, Wells D, Cybulska C, Liss J, Plociennik L, Kuczynski W, Zabielska J (2015) Routine use of next-generation sequencing for preimplantation genetic diagnosis of blastomeres obtained from embryos on day 3 in fresh in vitro fertilization cycles. Fertility and sterility 103 (4):1031-1036. doi:10.1016/j.fertnstert.2014.12.123

23. Popovic M, Dheedene A, Christodoulou C, Taelman J, Dhaenens L, Van Nieuwerburgh F, Deforce D, Van den Abbeel E, De Sutter P, Menten B, Heindryckx B (2018) Chromosomal mosaicism in human blastocysts: the ultimate challenge of preimplantation genetic testing? Human reproduction 33 (7):1342-1354. doi:10.1093/humrep/dey106

24. Spinella F, Fiorentino F, Biricik A, Bono S, Ruberti A, Cotroneo E, Baldi M, Cursio E, Minasi MG, Greco E (2018) Extent of chromosomal mosaicism influences the clinical outcome of in vitro fertilization treatments. Fertility and sterility 109 (1):77-83. doi:10.1016/j.fertnstert.2017.09.025

25. Fragouli E, Alfarawati S, Spath K, Babariya D, Tarozzi N, Borini A, Wells D (2017) Analysis of implantation and ongoing pregnancy rates following the transfer of mosaic diploid-aneuploid blastocysts. Human genetics 136 (7):805-819. doi:10.1007/s00439-017-1797-4

26. Greco E, Minasi MG, Fiorentino F (2015) Healthy Babies after Intrauterine Transfer of Mosaic Aneuploid Blastocysts. The New England journal of medicine 373 (21):2089-2090. doi:10.1056/NEJMc1500421

27. Kushnir VA, Darmon SK, Barad DH, Gleicher N (2018) Degree of mosaicism in trophectoderm does not predict pregnancy potential: a corrected analysis of pregnancy outcomes following transfer of mosaic embryos. Reproductive biology and endocrinology : RB\&E 16 (1):6. doi:10.1186/s12958-018$0322-5$

28. Tobler KJ, Brezina PR, Benner AT, Du L, Xu X, Kearns WG (2014) Two different microarray technologies for preimplantation genetic diagnosis and screening, due to reciprocal translocation imbalances, demonstrate equivalent euploidy and clinical pregnancy rates. Journal of assisted reproduction and genetics 31 (7):843-850. doi:10.1007/s10815-014-0230-3

29. Ou Z, Chen Z, Yin M, Deng Y, Liang Y, Wang W, Yao Y, Sun L (2020) Re-analysis of whole blastocysts after trophectoderm biopsy indicated chromosome aneuploidy. Human genomics 14 (1):3. doi:10.1186/s40246-019-0253-z

\section{Figures}


Figure 1
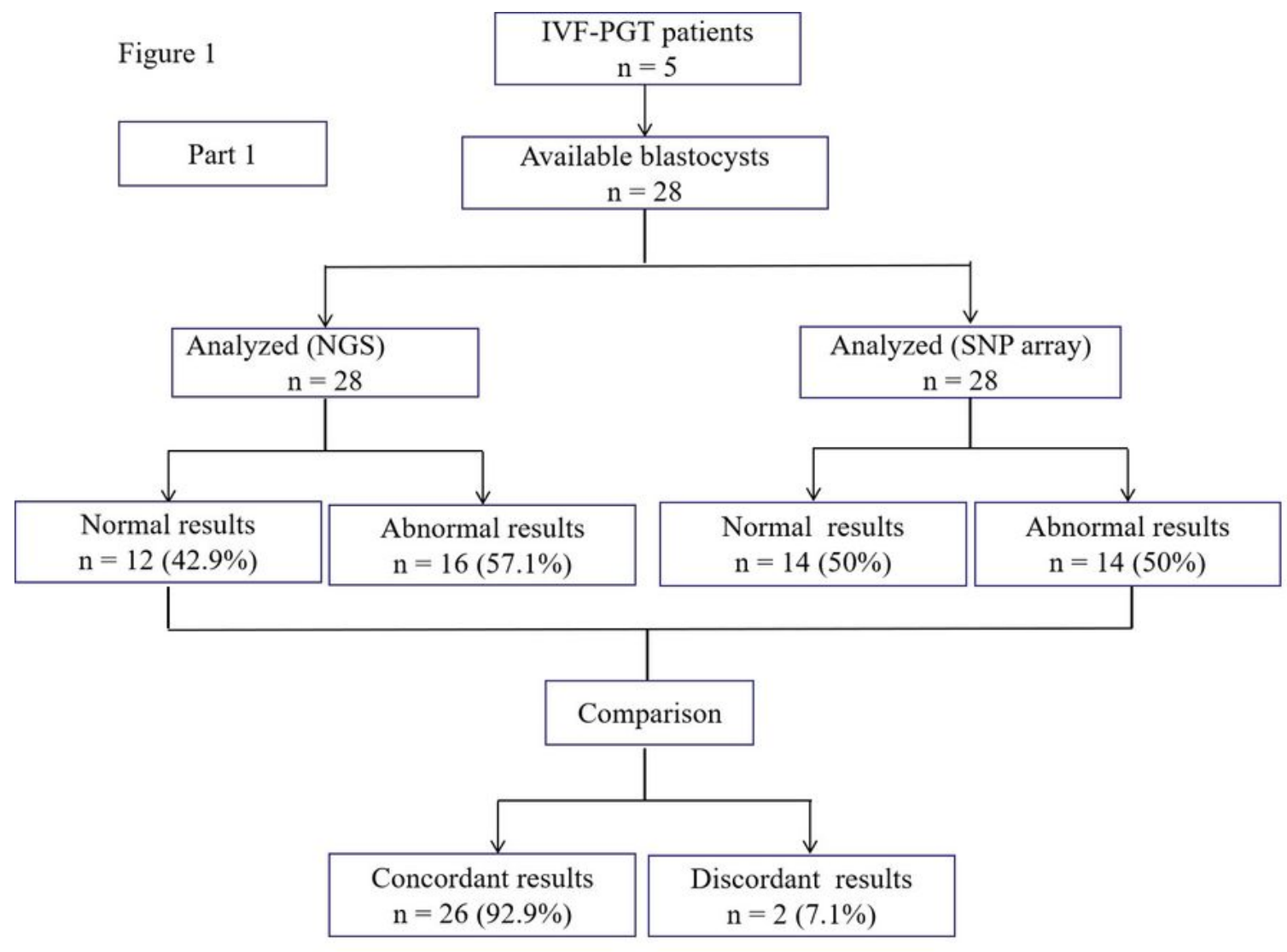

Part 2

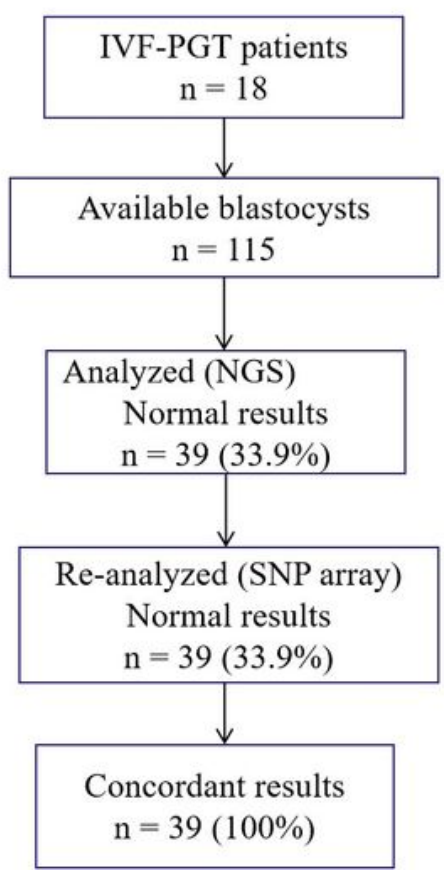

\section{Figure 1}

The flow diagram of this study. 

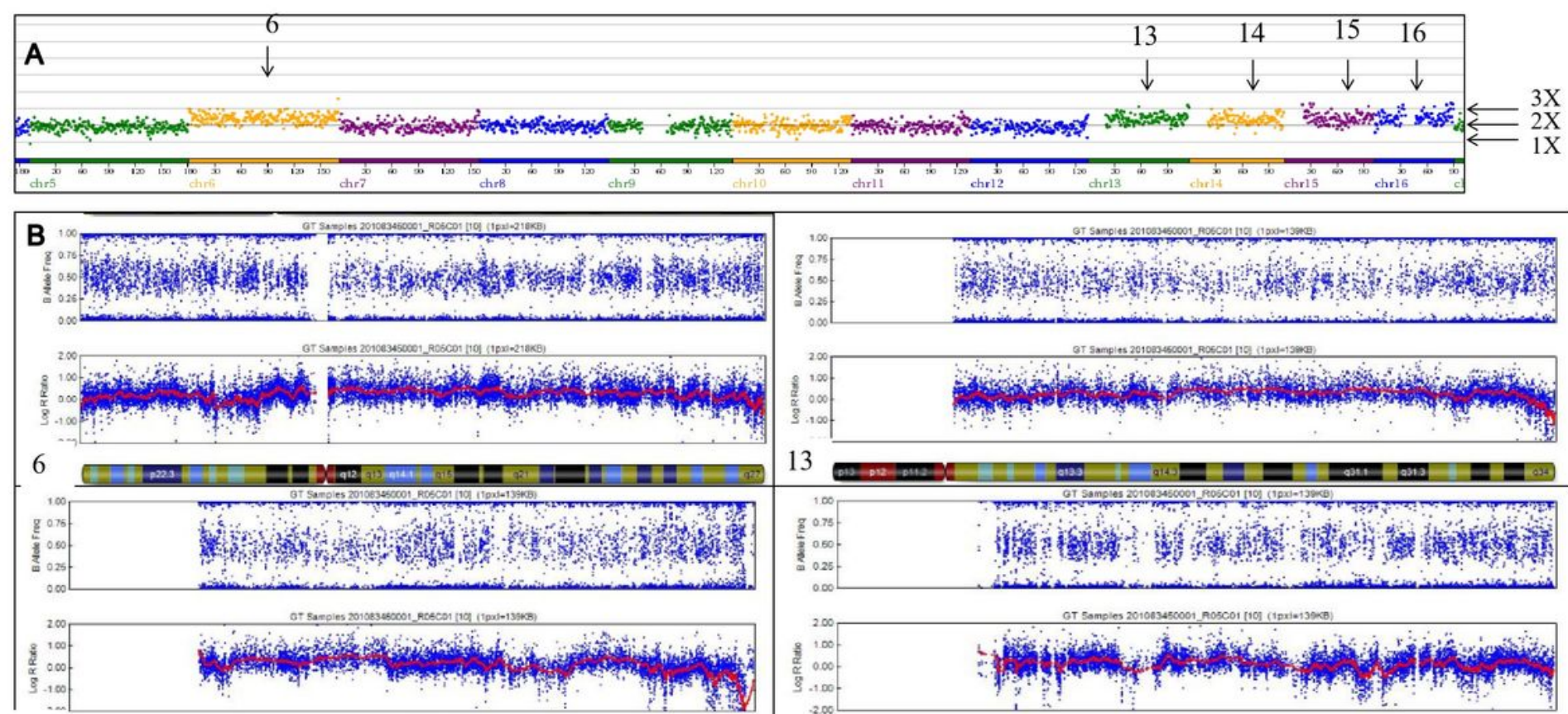

14
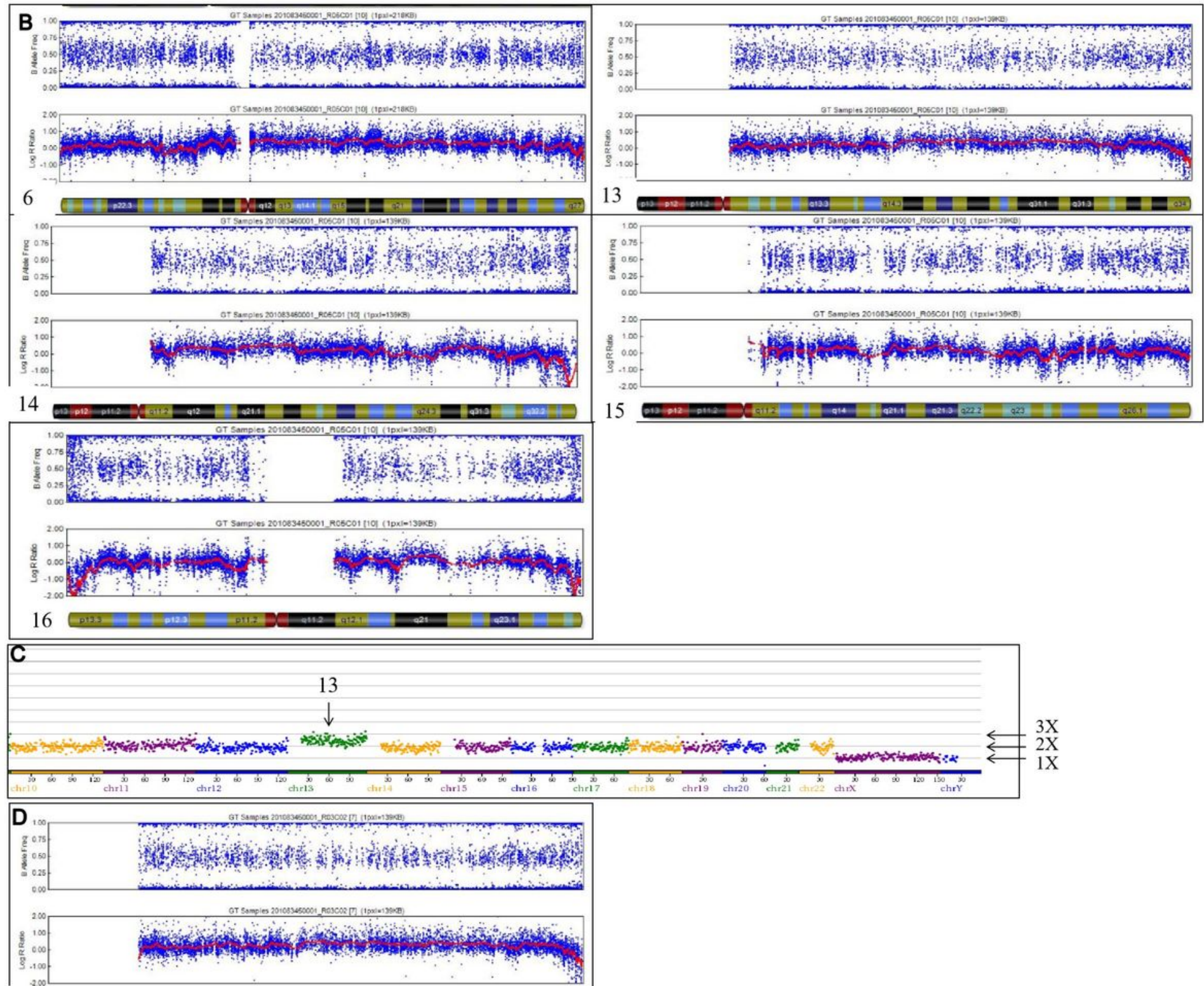

13

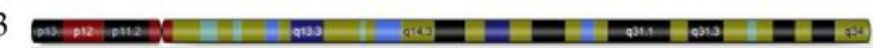

Figure 2

The discordant results of two blastocyst detected by NGS and SNP array. A. The abnormality detected by NGS with low level of mosaicism in the chromosome 6,13, 14, 15 and 16. 2X: the chromosome with two copy (normal), 3X: three copy, 1X: one copy. B. The normal result detected by SNP array in the chromosome $6,13,14,15$ and 16 . C. The abnormality detected by NGS with low level of mosaicism in the chromosome 13. 2X: the chromosome with two copy (normal), 3X: three copy, 1X: one copy. D. The normal result detected by SNP array in the chromosome 13. 


\section{Figure 3}
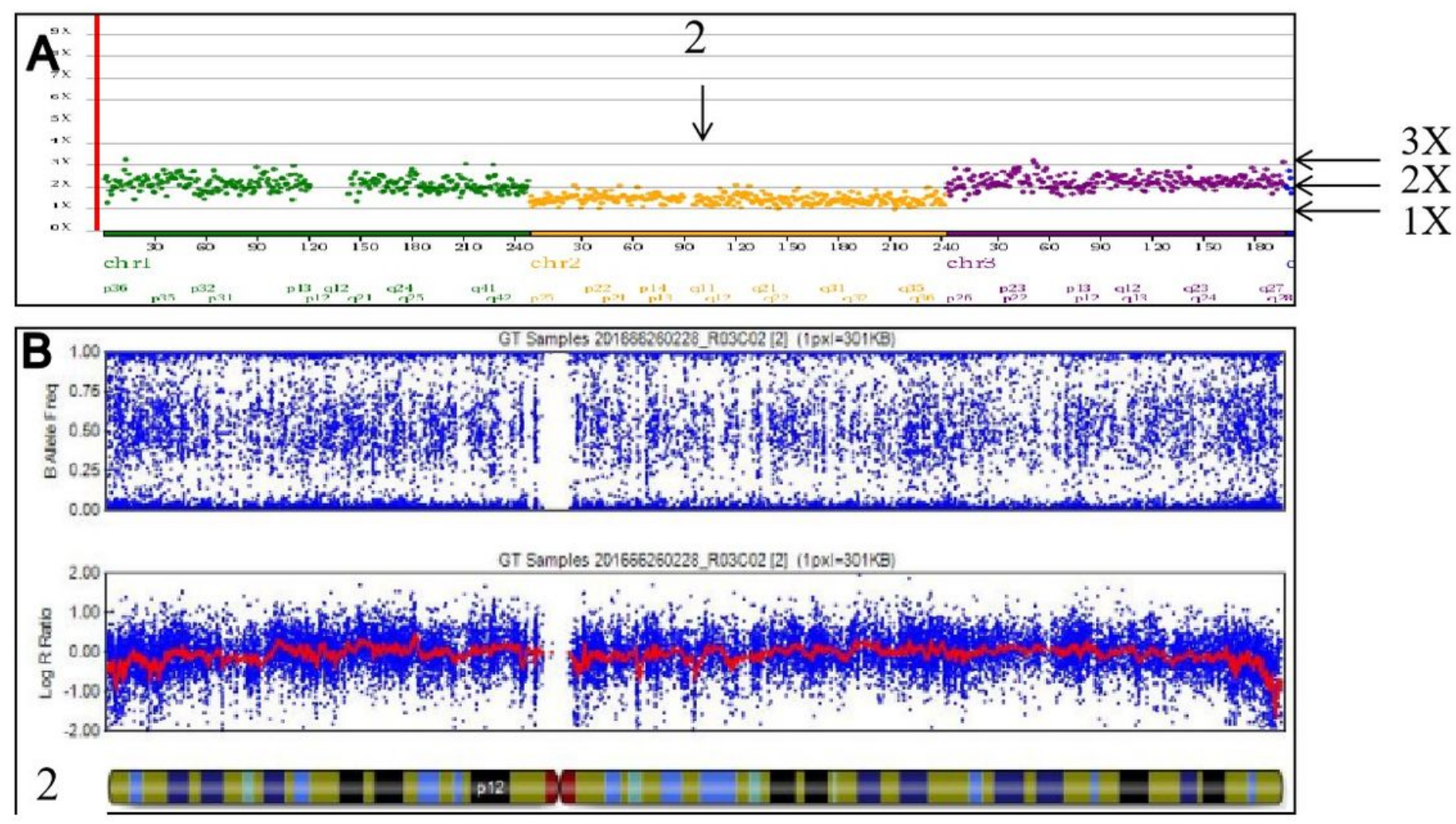

Figure 3

The discordant results of single blastocyst detected by NGS and SNP array. A. The abnormality detected by NGS with low level of mosaicism in the chromosome. 2X: the chromosome with two copy (normal), 3X: three copy, 1X: one copy. 2. B. The normal result detected by SNP array in the chromosome 2. 